\title{
Commentary: Large Acid-Evoked Currents, Mediated by ASIC1a, Accompany Differentiation in Human Dopaminergic Neurons
}

\author{
Matthew William, Som Singh and Xiang-Ping Chu* \\ Department of Biomedical Sciences, School of Medicine, University of Missouri-Kansas City, Kansas City, MO, United States
}

Keywords: acid-sensing ion channel, dopamine cell, differentiation, patch-clamp recording, human cell

\section{A Commentary on}

Large Acid-Evoked Currents, Mediated by ASIC1a, Accompany Differentiation in Human Dopaminergic Neurons

by Neuhof, A., Tian, Y., Reska, A., Falkenburger, B. H., and Gründer, S. (2021). Front. Cell. Neurosci. 15:668008. doi: 10.3389/fncel.2021.668008

\section{INTRODUCTION}

OPEN ACCESS

Edited by:

Patricia Armati,

The University of Sydney, Australia

Reviewed by:

Cheng-Chang Lien,

National Yang-Ming University, Taiwan

*Correspondence:

Xiang-Ping Chu

chux@umkc.edu

Specialty section:

This article was submitted to

Cellular Neurophysiology,

a section of the journa

Frontiers in Cellular Neuroscience

Received: 04 October 2021

Accepted: 29 October 2021

Published: 22 November 2021

Citation:

William M, Singh S and Chu X-P (2021) Commentary: Large

Acid-Evoked Currents, Mediated by ASIC1a, Accompany Differentiation in Human Dopaminergic Neurons. Front. Cell. Neurosci. 15:789354. doi: 10.3389/fncel.2021.789354
Acid-sensing ion channels (ASICs) are proton-gated $\mathrm{Na}^{+}$channels widely located in the peripheral and central nervous system (Waldmann et al., 1997; Krishtal, 2015). They are often associated with neurological disorders involving a decrease in extracellular $\mathrm{pH}$, such as cerebral ischemia and epilepsy (Xiong et al., 2008; Kweon and Suh, 2013; Li et al., 2016). Additionally, these channels also contribute to cognitive functions such as learning, memory and synaptic plasticity (Chu and Xiong, 2012; Huang et al., 2015; Gobetto et al., 2021). While the functions of ASICs are often explored in murine models, few studies have investigated these channels in human cells and human cell lines (Sun et al., 2020; Xu et al., 2021). Recently, a study conducted by the Grunder laboratory has examined the function of ASICs in human dopaminergic cell lines (Neuhof et al., 2021). They have proposed that ASICs are responsible for the differentiation of LUHMES (Lund Human Mesencephalic) cells, a line of human cells capable of differentiating into dopaminergic-like neurons (Zuberek et al., 2018; Harischandra et al., 2019). LUHMES cells are often used as a model to investigate cellular mechanisms of dopaminergic neurons in the substantia nigra (SN) and are used to further understand the pathology of disorders involving these neurons, such as Parkinson's disease (PD) (Zhang et al., 2014; Smirnova et al., 2016; Tüshaus et al., 2021). Their results ultimately reveal LUHMES cells to be a promising model in studying the role of ASICs in neuronal differentiation.

\section{FUNCTIONAL ASICS MODULATE THE DIFFERENTIATION OF LUHMES CELLS}

A recent study published in Frontiers in Cellular Neuroscience from the Grunder laboratory has explored the role of ASICs in the differentiation of LUHMES cells (Neuhof et al., 2021). They first converted LUHMES cells into post-mitotic dopaminergic neurons and subsequently isolated ASIC mRNA. They found that ASIC2 mRNA expression was profoundly lower than expression of ASIC1a mRNA, suggesting that ASIC1a is the dominant variant in LUHMES cells. Next, they measured 
the ASICla protein quantity and found that it was twice as high after 3 days of differentiation, however, any further increase in ASIC1a protein was halted after that. Whole-cell patch-clamp recording was used to record the ASIC currents. Peak ASIC current density started to increase significantly from day 0 to day 5 , and then began to decrease over the next 5 days. These results demonstrate that a strong surge in ASIC current density exists within the first 5 days of LUHMES cell differentiation, confirming the presence of functional ASICs. Furthermore, the presence of homomeric ASIC1a in this cell line was largely examined by measuring ASIC current amplitude. Half maximal activation of ASICs was detected at $\mathrm{pH} 6.6$ on day 4 and day 7 and $\mathrm{pH} 6.5$ on day 5 and day 6 , respectively, with a saturating amplitude of $\mathrm{pH}$ 6.0. These results strongly suggest that homomeric ASIC1a is likely responsible for the surge in ASIC current. Application of PcTx1, a selective ASIC1a inhibitor, resulted in complete inhibition of ASIC currents, further confirming the presence of homomeric ASIC1a. The presence of heteromeric ASIC1a/2b, however, cannot be excluded due to its similarities with homomeric ASIC1a, such as PcTx1 sensitivity. The presence of heteromeric ASIC1a/2a was not confirmed. Whether activation of ASICs can elicit action potentials (APs) in differentiating LUHMES cells was further investigated. At day 0, no APs were elicited upon depolarization, however, APs were elicited on day 5 and later after $\mathrm{pH}$ drop. The number of cells able to elicit APs nearly doubled from day 5 to day 10, indicating an increase in the excitability of LUHMES cells during the differentiation process. The role of ASICs in the physiological growth of LUHMES cells was further examined. After the ASIC pore blocker diminazene was applied, the length of neurites doubled from day 1 to day 5 of differentiation in a control population, while the diminazene-treated population contained shorter neurites on all days investigated. These results suggest that ASICs play a critical role in the neuronal differentiation process. Finally, the permeability of $\mathrm{Ca}^{2+}$ through ASICs was detected by using Fura-2 dye. Activation of ASICs by $\mathrm{pH}$ drop results in strong intracellular $\mathrm{Ca}^{2+}$ signaling. Upon application of the ASIC blocker amiloride, the $\mathrm{Ca}^{2+}$ response was nearly completely inhibited. While inhibition of voltagegated $\mathrm{Ca}^{2+}$ channels by nimodipine was found to reduce $\mathrm{Ca}^{2+}$ signaling as well, it was not as strong as the reduction seen with the application of amiloride. Thus, activation of ASICs in LUHMES cells plays a dominant role in the intracellular $\mathrm{Ca}^{2+}$ signaling response.

\section{DISCUSSION}

The study conducted by the Grunder laboratory provides evidence supporting the role of ASIC1a in the $\mathrm{Ca}^{2+}$ homeostasis of LUHMES cells undergoing differentiation. As alluded to in the study, dysregulation in $\mathrm{Ca}^{2+}$ homeostasis is a contributing factor to the development of neurodegenerative disorders of dopaminergic neurons, such as $\mathrm{PD}$. Oxidative stress is another risk factor involved in PD (Tabata et al., 2018; Trist et al., 2019). A recent study reported that ASICla was upregulated in
NS20Y cells with exposure to $\mathrm{H}_{2} \mathrm{O}_{2}$ for at least $6 \mathrm{~h}(\mathrm{Wu}$ et al., 2020). Thus, it is important to see whether the $\mathrm{H}_{2} \mathrm{O}_{2}$ treatment may upregulate ASIC1a expression in differentiated LUHMES cells. While $\mathrm{H}_{2} \mathrm{O}_{2}$ can induce oxidative damage to tissue which may also contribute to PD (Dehhaghi et al., 2018; Nandi et al., 2019), its role in potentially upregulating ASIC1a in dopaminergic neurons of the SN may contribute to overexpression of ASICla. The potential effects of this upregulation on calcium homeostasis in dopaminergic neurons may contribute to the calcium dysregulation hypothesized to cause PD (Mattson and Arumugam, 2018; Schrank et al., 2020). Therefore, investigation of the influence of $\mathrm{H}_{2} \mathrm{O}_{2}$ exposure on ASIC upregulation should be conducted to examine changes in intracellular calcium in LUHMES cells and cerebral dopaminergic neurons. Another avenue to explore is the limitations created by diminazene-treated LUHMES cells. While these cells have been shown to display physiological limitations, such as decreased neurite length by inhibition of ASICs, the ability to elicit APs or $\mathrm{Ca}^{2+}$ signals should also be investigated under ASIC blockers. If diminazene-treated cells with shorter neurites possess diminished or decreased ability to elicit APs or $\mathrm{Ca}^{2+}$ signals, this could provide further support for the importance of ASICs in LUHMES cell differentiation. A third avenue to investigate is the role of ASICs in disorders involving an increase in dopamine release, such as schizophrenia. The paper published by the Grunder laboratory suggests that ASIC1a may modulate dopamine secretion from human dopaminergic neurons (Neuhof et al., 2021). Schizophrenia is hypothesized to occur due to an over secretion of dopamine in the mesolimbic pathway (Birtwistle and Baldwin, 1998; Jones et al., 2011; Howes et al., 2017). Therefore, investigating ASIC1a in dopaminergic neurons of the mesolimbic pathway as a potential therapeutic target in limiting positive schizophrenic symptoms resulting from the over secretion of dopamine may prove clinically useful.

\section{AUTHOR CONTRIBUTIONS}

All authors listed have made a substantial, direct and intellectual contribution to the work, and approved it for publication.

\section{FUNDING}

The work was supported by grant from American Heart Association (19AIREA34470007) to X-PC.

\section{ACKNOWLEDGMENTS}

We would like to thank the University of Missouri-Kansas City School of Medicine student research program for their support of MW and SS's professional studies. MW and SS are Sarah Morrison Student Research Award recipient by the University of Missouri-Kansas City School of Medicine. 


\section{REFERENCES}

Birtwistle, J., and Baldwin, D. (1998). Role of dopamine in schizophrenia and Parkinson's disease. Br. J. Nurs. 7, 832-4, 836, 838-841. doi: 10.12968/bjon.1998.7.14.5636

Chu, X. P., and Xiong, Z. G. (2012). Physiological and pathological functions of acid-sensing ion channels in the central nervous system. Curr. Drug Targets 13, 263-271. doi: 10.2174/138945012799201685

Dehhaghi, M., Tan, V., Heng, B., Mohammadipanah, F., and Guillemin, G. J. (2018). Protective effects of myxobacterial extracts on hydrogen peroxideinduced toxicity on human primary astrocytes. Neuroscience 399, 1-11. doi: 10.1016/j.neuroscience.2018.11.033

Gobetto, M. N., González-Inchauspe, C., and Uchitel, O. D. (2021). Histamine and corticosterone modulate acid sensing ion channels (ASICs) dependent longterm potentiation at the mouse anterior cingulate cortex. Neuroscience 460, 145-160. doi: 10.1016/j.neuroscience.2021.01.022

Harischandra, D. S., Rokad, D., Ghaisas, S., Verma, S., Robertson, A., Jin, H., et al. (2019). Enhanced differentiation of human dopaminergic neuronal cell model for preclinical translational research in Parkinson's disease. Biochim. Biophys. Acta Mol. Basis Dis. 1866:165533. doi: 10.1016/j.bbadis.2019.165533

Howes, O. D., McCutcheon, R., Owen, M. J., and Murray, R. M. (2017). The role of genes, stress, and dopamine in the development of schizophrenia. Biol. Psychiatry 81, 9-20. doi: 10.1016/j.biopsych.2016.07.014

Huang, Y., Jiang, N., Li, J., Ji, Y. H., Xiong, Z. G., and Zha, X. M. (2015). Two aspects of ASIC function: synaptic plasticity and neuronal injury. Neuropharmacology 94, 42-48. doi: 10.1016/j.neuropharm.2014.12.010

Jones, C. A., Watson, D. J., and Fone, K. C. (2011). Animal models of schizophrenia. Br. J. Pharmacol. 164, 1162-1194. doi: 10.1111/j.1476-5381.2011.01386.x

Krishtal, O. (2015). Receptor for protons: first observations on acid sensing ion channels. Neuropharmacology 94, 4-8. doi: 10.1016/j.neuropharm.2014.12.014

Kweon, H. J., and Suh, B. C. (2013). Acid-sensing ion channels (ASICs): therapeutic targets for neurological diseases and their regulation. BMB Rep. 46, 295-304. doi: 10.5483/BMBRep.2013.46.6.121

Li, M. H., Leng, T. D., Feng, X. C., Yang, T., Simon, R. P., and Xiong, Z. G. (2016). Modulation of acid-sensing ion channel 1a by intracellular $\mathrm{pH}$ and its role in ischemic stroke. J. Biol. Chem. 291, 18370-18383. doi: 10.1074/jbc.M115.713636

Mattson, M. P., and Arumugam, T. V. (2018). Hallmarks of brain aging: adaptive and pathological modification by metabolic states. Cell Metab. 27, 1176-1199. doi: 10.1016/j.cmet.2018.05.011

Nandi, A., Yan, L. J., Jana, C. K., and Das, N. (2019). Role of catalase in oxidative stress- and age-associated degenerative diseases. Oxid. Med. Cell Longev. 2019:9613090. doi: 10.1155/2019/9613090

Neuhof, A., Tian, Y., Reska, A., Falkenburger, B. H., and Gründer, S. (2021). Large acid-evoked currents, mediated by ASIC1a, accompany differentiation in human dopaminergic neurons. Front. Cell. Neurosci. 15:668008. doi: 10.3389/fncel.2021.668008

Schrank, S., Barrington, N., and Stutzmann, G. E. (2020). Calcium-handling defects and neurodegenerative disease. Cold Spring Harb. Perspect. Biol. 12:a035212. doi: 10.1101/cshperspect.a035212

Smirnova, L., Harris, G., Delp, J., Valadares, M., Pamies, D., Hogberg, H. T., et al. (2016). A LUHMES 3D dopaminergic neuronal model for neurotoxicity testing allowing long-term exposure and cellular resilience analysis. Arch. Toxicol. 90, 2725-2743. doi: 10.1007/s00204-015-1637-z
Sun, D., Liu, S., Li, S., Zhang, M., Yang, F., Wen, M., et al. (2020). Structural insights into human acid-sensing ion channel 1a inhibition by snake toxin mambalgin1. Elife 9:e57096. doi: 10.7554/eLife.5 7096

Tabata, Y., Imaizumi, Y., Sugawara, M., et al. (2018). T-type calcium channels determine the vulnerability of dopaminergic neurons to mitochondrial stress in familial Parkinson's disease. Stem Cell Reports 11, 1171-1184. doi: 10.1016/j.stemcr.2018.0 9.006

Trist, B. G., Hare, D. J., and Double, K. L. (2019). Oxidative stress in the aging substantia nigra and the etiology of Parkinson's disease. Aging Cell 18:e13031. doi: 10.1111/acel.13031

Tüshaus, J., Kataka, E. S., Zaucha, J., Frishman, D., Müller, S. A., and Lichtenthaler, S. F. (2021). Neuronal differentiation of LUHMES cells induces substantial changes of the proteome. Proteomics 21:e2000174. doi: $10.1002 /$ pmic. 202000174

Waldmann, R., Champigny, G., Bassilana, F., Heurteaux, C., and Lazdunski, M. (1997). A proton-gated cation channel involved in acid-sensing. Nature 386, 173-177. doi: 10.1038/386173a0

Wu, B. M., Bargaineer, J., Zhang, L., Yang, T., Xiong, Z. G., and Leng, T. D. (2020). Upregulation of acid sensing ion channel la (ASIC1a) by hydrogen peroxide through the JNK pathway. Acta Pharmacol. Sin. 42, 1248-1255. doi: 10.1038/s41401-020-00559-3

Xiong, Z. G., Pignataro, G., Li, M., Chang, S. Y., and Simon, R. P. (2008). Acidsensing ion channels (ASICs) as pharmacological targets for neurodegenerative diseases. Curr. Opin. Pharmacol. 8, 25-32. doi: 10.1016/j.coph.2007.09.001

Xu, Y., Jiang, Y. Q., Li, C., et al. (2021). Human ASIC1a mediates stronger acidinduced responses as compared with mouse ASIC1a. FASEB J. 32, 3832-3843. doi: 10.1096/fj.201701367R

Zhang, X. M., Yin, M., and Zhang, M. H. (2014). Cell-based assays for Parkinson's disease using differentiated human LUHMES cells. Acta. Pharmacol. Sin. 35, 945-956. doi: 10.1038/aps.2014.36

Zuberek, M., Stepkowski, T. M., Kruszewski, M., and Grzelak, A. (2018). Exposure of human neurons to silver nanoparticles induces similar pattern of $\mathrm{ABC}$ transporters gene expression as differentiation: study on proliferating and post-mitotic LUHMES cells. Mech. Ageing Dev. 171, 7-14. doi: 10.1016/j.mad.2018.02.004

Conflict of Interest: The authors declare that the research was conducted in the absence of any commercial or financial relationships that could be construed as a potential conflict of interest.

Publisher's Note: All claims expressed in this article are solely those of the authors and do not necessarily represent those of their affiliated organizations, or those of the publisher, the editors and the reviewers. Any product that may be evaluated in this article, or claim that may be made by its manufacturer, is not guaranteed or endorsed by the publisher.

Copyright (C) 2021 William, Singh and Chu. This is an open-access article distributed under the terms of the Creative Commons Attribution License (CC BY). The use, distribution or reproduction in other forums is permitted, provided the original author(s) and the copyright owner(s) are credited and that the original publication in this journal is cited, in accordance with accepted academic practice. No use, distribution or reproduction is permitted which does not comply with these terms. 